EPJ Web of Conferences 49, 15017 (2013)

DOI: 10.1051/epjconf/20134915017

(C) Owned by the authors, published by EDP Sciences, 2013

\title{
LHC Signals of Pure Gravity Mediation
}

\author{
Brian Feldstein ${ }^{1, a}$ \\ ${ }^{1}$ Kavli IPMU, University of Tokyo, Kashiwa, 277-8583, Japan
}

\begin{abstract}
Evidence is mounting that natural supersymmetry at the weak scale is not realized in nature. This evidence comes from collider searches, a lack of new flavor changing neutral current effects, and now also the size of the measured Higgs mass. On the other hand, string theory suggests that supersymmetry might be present at some energy scale, and gauge coupling unification and dark matter imply that that energy scale may be relatively low. The simplest model to address all of these hints is arguably "pure gravity mediation", in which the scalar superpartner masses are taken to be perhaps $100 \mathrm{TeV}$, with gauginos automatically acquiring loop factor suppressed masses of order TeV. The gauginos might then be the only superpartners accessible to the LHC. Unification and LSP dark matter are maintained (with a wino LSP) at the cost of a $10^{-5}$ or $10^{-6}$ fine tuning. Here I will discuss the structure and LHC phenomenology of pure gravity mediation.
\end{abstract}

\section{Introduction}

Models of natural weak scale supersymmetry face increasing challenges. These include:

- The apparent observation of a Higgs particle with mass of $\sim 125 \mathrm{GeV}-$ heavier than what may normally be obtained in simple supersymmetric models. Indeed, at tree level in the minimal supersymmetric standard model (MSSM), the Higgs mass is bounded to be less than the $\mathrm{Z}$ boson mass.

- The lack of any direct observation of superpartners. Naively, superpartners should have been detected by now in order to avoid having to fine-tune to obtain appropriate masses for the $\mathrm{W}$ and $\mathrm{Z}$ bosons.

- The consistency of observed flavor changing neutral current (FCNC) effects with the predictions of the standard model. Even before the LHC, this provided a severe constraint on the possible structure of the superpartner masses.

In spite of this, supersymmetry remains an attractive framework for physics beyond the standard model. As well as improving the naturalness of the theory, weak scale supersymmetry results in gauge coupling unification, and- with the assumption of R-parity conservationyields a dark matter candidate. In addition, it is interesting that string theory appears to require supersymmetry to be present at some scale.

Here we will emphasize that if one drops the requirement of strict naturalness of the weak scale, then perhaps the simplest supersymmetric scenario becomes viablethat of "pure gravity mediation" [1-6]- and all of the phenomenological difficulties noted above may be solved. At the same time, the lightest superpartner (LSP) can still be

\footnotetext{
a e-mail: brian.feldstein@ipmu.jp
}

a dark matter candidate, and gauge coupling unification remains successful.

In pure gravity mediation, one assumes that supersymmetry is broken by the vacuum expectation value of the F-term $F_{Z}$ of a chiral superfield $Z$ in a hidden sector. The scalar superpartners, as well as the gravitino, then obtain masses automatically via gravitational interactions of order $m_{\text {susy }} \sim F_{Z} / M_{\mathrm{pl}}$. The higgsino masses depend on the mechanism for solving the $\mu$ problem, but may be perhaps most simply taken also of order $m_{\text {susy }}$, via the GiudiceMasiero mechanism [7]. Solving the FCNC problem without any additional assumptions then requires $m_{\text {susy }} \gtrsim 1000$ TeV. A minor flavor symmetry, or fortuitously somewhat suppressed CP violating contribution to $K^{0}-\bar{K}^{0}$ mixing can also allow for $m_{\text {susy }}$ of perhaps order $100 \mathrm{TeV}$. This is to be contrasted with the case of natural weak scale supersymmetry, in which off diagonal squark mass matrix elements must be suppressed by perhaps four orders of magnitude. Superpartner masses of order $100-1000 \mathrm{TeV}$ also automatically raise the Higgs mass up to an appropriate size via radiative corrections, as can be seen in figure 1 . Constraints from collider searches performed to date may also be avoided.

The key question concerning pure gravity mediation then becomes one of testability; while the superpartners at the scale $m_{\text {susy }}$ are clearly out of reach of planned colliders, this need not also be the case for the gauginos: If the supersymmetry breaking field $Z$ is charged under any kind of symmetry (as is motivated in fact, by the Polonyi problem [8]), then it is impossible for the gauginos to also obtain masses directly at order $F_{Z} / M_{\mathrm{pl}}$. In fact, the gauginos obtain $O(\mathrm{TeV})$ masses suppressed compared to $F_{Z} / M_{\mathrm{pl}}$ by a loop factor of $10^{-3}-10^{-2}$, and may thus be accessible to the LHC. In detail, the gauginos obtain masses via anomaly mediation and higgsino threshold corrections [9- 


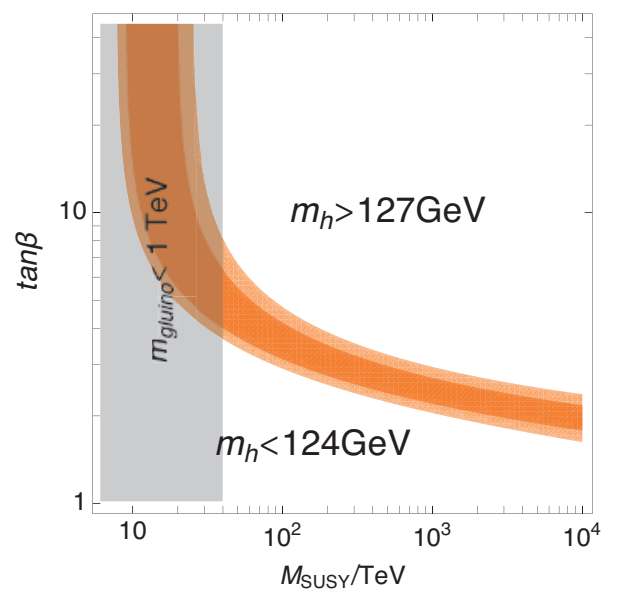

Figure 1. The contour plot of the lightest Higgs boson mass. Here, we have fixed $m_{3 / 2}=\mu_{H}=M_{\text {SusY }}$. The orange band shows the Higgs boson mass $124 \mathrm{GeV}<m_{h}<127 \mathrm{GeV}$ observed by the ATLAS and CMS collaborations for the central value of the top quark mass. The light orange band is the one including the $1 \sigma$ error of the top quark mass. In the gray shaded region, the gluino mass is below about $1 \mathrm{TeV}$, as predicted by anomaly mediation with $M_{\text {SUSY }}=m_{3 / 2}$ (see equation (1)).

11], and their spectrum takes the form [3]:

$$
\begin{gathered}
m_{\text {gluino }} \simeq 2.5 \times\left(1-0.13 \delta_{32}-0.04 \delta_{\text {SUSY }}\right) \times 10^{-2} m_{3 / 2}, \\
m_{\text {wino }} \simeq 3.0 \times\left(1-0.04 \delta_{32}+0.02 \delta_{\text {SUSY }}\right) \times 10^{-3}\left(m_{3 / 2}+L\right), \\
m_{\text {bino }} \simeq 9.6 \times\left(1+0.01 \delta_{\text {SUSY }}\right) \times 10^{-3}\left(m_{3 / 2}+L / 11\right),
\end{gathered}
$$

where $\delta_{\text {SUSY }}=\log \left[M_{\text {SUSY }} / 100 \mathrm{TeV}\right], \delta_{32}$ denotes $\delta_{32}=$ $\log \left[m_{3 / 2} / 100 \mathrm{TeV}\right]$ for the gluino, and $\delta_{32}=\log \left[\left(m_{3 / 2}+\right.\right.$ $L) / 100 \mathrm{TeV}]$ for the wino. The terms proportional to $m_{3 / 2}$ in the above formulae represent the anomaly mediated contributions, while those proportional to $L$ are the higgsino threshold contributions, with $L$ given by

$$
L \equiv \mu_{H} \sin 2 \beta \frac{m_{A}^{2}}{\left|\mu_{H}\right|^{2}-m_{A}^{2}} \ln \frac{\left|\mu_{H}\right|^{2}}{m_{A}^{2}} .
$$

As discussed in references [1,2], $L$ is expected to be of the order of the gravitino mass. The wino mass therefore obtains comparable contributions from both the anomaly mediated effects and the higgsino threshold corrections.

In what follows, we will discuss the LHC phenomenology of the gauginos, restricting ourselves to the case of a wino LSP in order to obtain an appropriate dark matter candidate.

\section{LHC Phenomenology}

Of relevance to collider searches is the small splitting between the charged and neutral components of the wino, which is of order [12]

$$
\Delta m_{\text {wino }} \simeq 160-170 \mathrm{MeV} .
$$

As a result, the neutral and the charged wino are highly degenerate and the dominant decay mode of the charged wino is into a neutralino and a charged pion, $\tilde{\chi}^{ \pm} \rightarrow \tilde{\chi}^{0}+\pi^{ \pm}$. The charged wino has a rather long lifetime for the purpose of collider physics,

$$
\tau_{\text {wino }} \simeq 1.4 \times 10^{-10} \sec \left(\frac{160 \mathrm{MeV}}{\Delta m_{\text {wino }}}\right)^{3}\left(1-\frac{m_{\pi}^{2}}{\Delta m_{\text {wino }}^{2}}\right)^{-1 / 2},
$$

and any charged winos produced at the LHC experiment will typically travel $O(1-10) \mathrm{cm}$ before decaying. ${ }^{1}$ As a result, it is possible to try to reduce standard model backgrounds by looking for events with a disappearing charged track; the charged pion in the final state of the decay has little energy and is not detected. LHC searches may thus be divided into several classes: Those in which the gluino is or isn't light enough to be appreciably produced at the LHC, and those in which one does or doesn't require the observation of a disappearing charged track.

We first consider the present bounds which may be obtained from gluino pair production without use of disappearing track information. The event topology of the signal is multiple jets with large missing momentum, this being one of the typical SUSY signals studied well by both the ATLAS and CMS collaborations. Results of a calculation of the cross section (times acceptance) for various gluino and wino masses were compared to the experimental upper limit shown in reference [14]. We thereby obtained the bounds on the gluino and wino masses at $95 \%$ confidence level shown in figure 2 by the solid blue line. It turns out that the region with $m_{\text {gluino }} \lesssim 1 \mathrm{TeV}$ and $m_{\text {wino }} \lesssim$ $300 \mathrm{GeV}$ has been excluded. For details of the analysis, please see [3]. Since the pattern of gluino decay depends on the details of the squark mass spectrum, and the bounds on the gluino and wino masses are not significantly different in any case, in these discussions we simply assume that all gluinos decay into two light quarks and a neutral/charged wino. We also assume to be explicit that onethird of gluinos decay into light quarks by emitting a neutral wino and the other two-thirds by emitting a charged wino.

We next consider the bounds from gluino pair production with use of the disappearing track information. We have utilized a result presented in reference [18], where model-independent upper limits on the cross section (times the acceptance) for non-SM physics events with an isolated disappearing track are provided. The resulting bounds on the gluino and wino masses are shown in figure 2 as a solid magenta line. Since the location of the TRT is $1 \mathrm{~m}$ away from the beam pipe and the typical decay length of the charged wino is $5 \mathrm{~cm}$, the bounds are weaker than those obtained by the conventional analysis (the gluino pair production without use of the disappearing track information). Note that, in particular, when the wino mass becomes heavier, the gamma-factor of the charged wino becomes smaller, which results in weaker limits on gluino production. If more inner detectors were used to look for disappearing tracks, on the other hand, the bounds would become much stronger, as will be discussed in the next section.

\footnotetext{
${ }^{1}$ For an updated two loop result, see [13]
} 


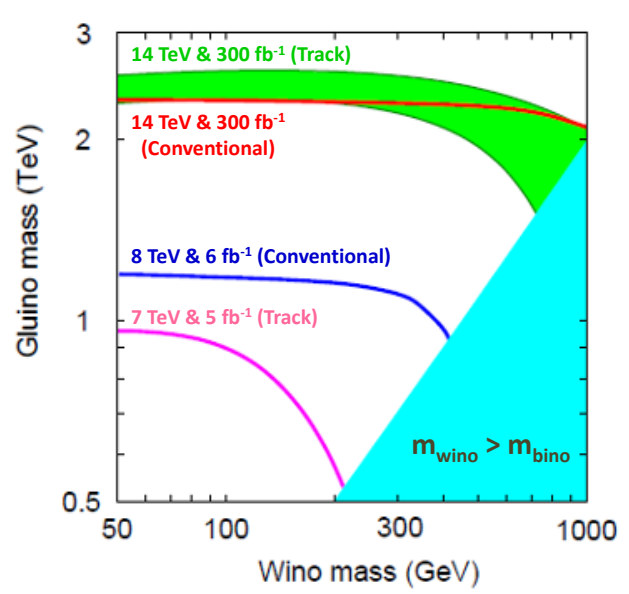

Figure 2. Present bounds and future reach in the gluino/wino mass plane in pure gravity mediation. The solid blue line was obtained via the gluino pair production process without use of the disappearing track information $\left(6 \mathrm{fb}^{-1}\right.$ of data at $\left.8 \mathrm{TeV}\right)$, while the solid magenta line was obtained with use of the disappearing track information $\left(5 \mathrm{fb}^{-1}\right.$ of data at $\left.7 \mathrm{TeV}\right)$. For future prospects, we show in red the reach of the search without disappearing track information ( $300 \mathrm{fb}^{-1}$ of data at $14 \mathrm{TeV}$ ), and in green the search with disappearing track information. The green curve actually forms a band, corresponding to choices for the parameter $\epsilon$ between 0 and 0.1 (see text). The region with $m_{\text {bino }}<m_{\text {wino }}$ is shaded because this region is not favored from the viewpoint of cosmology (overclosure of the universe).

Finally let us comment on the bound on the wino mass which is obtained from direct wino production with use of the disappearing track information. At the time this work was completed, the best official bound was that placed by the LEP experiment of $92 \mathrm{GeV}$ at $95 \%$ confidence level [20]. This constraint was obtained by searching for the radiative return process from wino pair production $\left(e^{+} e^{-} \rightarrow \gamma \tilde{\chi} \tilde{\chi}\right.$ with $\tilde{\chi}$ being a charged or a neutral wino). However, recently, a new limit of about $110 \mathrm{GeV}$ was obtained by ATLAS using $5 \mathrm{fb}^{-1}$ of data at $7 \mathrm{TeV}$. Please see [13, 19].

\section{Future Prospects}

As in the discussion in the previous subsection, we first consider the expected bounds on the gluino and wino masses obtained from the gluino pair production without use of the disappearing track information. We have assumed an accumulation of $300 \mathrm{fb}^{-1}$ of data at the $14 \mathrm{TeV}$ LHC, and utilized the result in reference [21] to estimate the SM backgrounds. The result of a calculation for the cross section (times acceptance) have been compared with the SM backgrounds in the reference and we then obtained the expected bounds at $95 \%$ confidence level, which are shown in figure 2 as the solid red line. The region with $m_{\text {gluino }} \lesssim 2.4 \mathrm{TeV}$ and $m_{\text {wino }} \lesssim 1 \mathrm{TeV}$ will be covered at the future LHC experiment.

We next consider the expected bounds on the gluino and wino masses from gluino pair production with the use of disappearing track information. SM backgrounds against this signal are, unfortunately, difficult to estimate without the use of real data. We have therefore introduced a parameter $\epsilon$ in order to describe how efficiently the SM backgrounds can be reduced when we impose selection criteria for the isolated and charged disappearing track. The cross section of the SM backgrounds is then estimated as $\epsilon$ times $\sigma_{\mathrm{BG}}$ with $\sigma_{\mathrm{BG}}$ being the background cross section without imposing the existence of the track. Results for the signal cross section for various gluino and wino masses have been compared with the resulting SM backgrounds to obtain the expected bounds at $95 \%$ confidence level, shown in figure 2 as the green band for the cases of $\epsilon$ between 0 and 0.1 . When the wino is relatively light and $\epsilon$ is small enough, this process gives stronger bounds than were obtained without looking for a disappearing track. In our analysis, we have assumed somewhat optimistic parameters for finding the track- namely, we are allowing the charged winos to decay between $142 \mathrm{~mm}$ and $520 \mathrm{~mm}$. If it is in fact necessary to use more outer detectors to find the track, then the bounds will be weaker as discussed in reference [22].

Finally we consider direct production of the wino through electroweak interactions at the future LHC experiment $\left(300 \mathrm{fb}^{-1} \& 14 \mathrm{TeV}\right)$. Since the cross section of the signal process is much smaller than that for gluino production, it is difficult to determine the expected bound on the wino mass without having accurate data for the SM backgrounds. We therefore briefly discuss some prospects of this process for constraining the PGM model using the signal cross section at parton level.

There are actually two production processes. One is the pair production of the wino associated with a jet [23, 24]. Based on the kinematical cuts in reference [25] to reduce QCD and electroweak backgrounds, we have calculated the cross section for wino production associated with a colored parton using the CalcHep code [26], which is shown in figure 3 as a solid magenta line. We also imposed selection cuts to obtain a charged track in the central region of the detector, the details of which may be found in [3]. The cross section of the SM backgrounds after applying the kinematical cuts (but before applying the selection cuts for the charged track) is about $700 \mathrm{fb}$.

A second process is the pair production of the wino through vector boson fusion (VBF). Based on the kinematical cuts in reference [27], which requires the existence of two forward jets (colored partons) with a large rapidity gap and a suppression of jet activities in the central region of the detector, we have calculated the cross section of the signal process using the CalcHep code, including again a requirement for a charged track in the central region of the detector. The result is shown in figure 3 as a solid blue line. The cross section for the VBF process is 5-10 times smaller than that for the wino pair production in association with a jet. The cross section of the SM backgrounds on the other hand is about $170 \mathrm{fb}$, which we note is much smaller than in the case of associated production. One of the attractive features of the use of the VBF process is that, since this process provides a clean environment at the central region of the detector, the charged disappearing track 


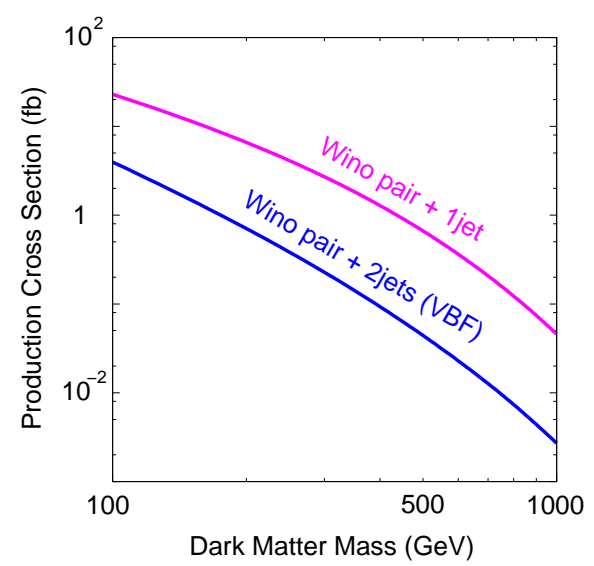

Figure 3. Cross sections for the direct wino production at the 14 $\mathrm{TeV} \mathrm{LHC}$ with $300 \mathrm{fb}^{-1}$ of data. The solid magenta line (wino pair +1 jet) is the wino pair production associated with a jet (a colored parton), while the solid blue line (wino pair +2 jets $(V B F))$ is the wino pair production through vector boson fusion processes. These cross sections are obtained after applying appropriate selection cuts. For details, please see the text.

will be efficiently searched for, though the absolute number of signal events is small.

The direct production of the wino is of importance to investigate the PGM model because it gives a bound on the wino mass independent of the gluino mass. Finding more sophisticated kinematical selections to increase the number of signal events and also, use of the most inner detectors, are therefore both ardently desired.

\section{Acknowledgments}

We would like to thank Shigeki Matsumoto for providing an updated figure. This work was supported by the World Premier International Research Center Initiative (WPI Initiative), MEXT, Japan.

\section{References}

[1] M. Ibe and T. T. Yanagida, Phys. Lett. B 709, 374 (2012).

[2] M. Ibe, S. Matsumoto and T. T. Yanagida, Phys. Rev. D 85, 095011 (2012).

[3] B. Bhattacherjee, B. Feldstein, M. Ibe, S. Matsumoto and T. T. Yanagida, arXiv:1207.5453 [hep-ph].

[4] L. J. Hall, Y. Nomura and S. Shirai, JHEP 1301, 036 (2013) [arXiv:1210.2395 [hep-ph]].

[5] A. Arvanitaki, N. Craig, S. Dimopoulos and G. Villadoro, arXiv:1210.0555 [hep-ph].
[6] N. Arkani-Hamed, A. Gupta, D. E. Kaplan, N. Weiner and T. Zorawski, arXiv:1212.6971 [hep-ph].

[7] G. F. Giudice and A. Masiero, Phys. Lett. B 206, 480 (1988).

[8] G. D. Coughlan, W. Fischler, E. W. Kolb, S. Raby and G. G. Ross, Phys. Lett. B 131, 59 (1983).

[9] G. F. Giudice, M. A. Luty, H. Murayama and R. Rattazzi, JHEP 9812, 027 (1998).

[10] L. Randall and R. Sundrum, Nucl. Phys. B 557, 79 (1999).

[11] M. Dine and D. MacIntire, Phys. Rev. D 46, 2594 (1992) [hep-ph/9205227].

[12] J. L. Feng, T. Moroi, L. Randall, M. Strassler and S. f. Su, Phys. Rev. Lett. 83, 1731 (1999).

[13] M. Ibe, S. Matsumoto and R. Sato, arXiv:1212.5989 [hep-ph].

[14] The ATLAS Collaboration, ATLAS-CONF-2012109.

[15] The CMS Collaboration, CMS PAS SUS-12-002; CMS PAS SUS-11-016.

[16] G. Aad et al. [ATLAS Collaboration], arXiv:1206.1760 [hep-ex]; The ATLAS Collaboration, ATLAS-CONF-2012-037.

[17] The ATLAS Collaboration, ATLAS-CONF-2012058.

[18] G. Aad et al. [ATLAS Collaboration], Eur. Phys. J. C 72, 1993 (2012); The ATLAS Collaboration, ATLASCONF-2012-034.

[19] [ATLAS Collaboration], JHEP 1301, 131 (2013) [arXiv:1210.2852 [hep-ex]].

[20] A. Heister et al. [ALEPH Collaboration], Phys. Lett. B 533, 223 (2002); G. Abbiendi et al. [OPAL Collaboration], Eur. Phys. J. C 29, 479 (2003); J. Abdallah et al. [DELPHI Collaboration], Eur. Phys. J. C 34, 145 (2004).

[21] S. Asai, T. Moroi, K. Nishihara and T. T. Yanagida, Phys. Lett. B 653, 81 (2007); S. Asai, T. Moroi and T. T. Yanagida, Phys. Lett. B 664, 185 (2008).

[22] G. Kane, R. Lu and B. Zheng, arXiv:1202.4448 [hepph].

[23] M. Ibe, T. Moroi and T. T. Yanagida, Phys. Lett. B 644, 355 (2007);

[24] T. Moroi and K. Nakayama, Phys. Lett. B 710, 159 (2012).

[25] M. Beltran, D. Hooper, E. W. Kolb, Z. A. C. Krusberg and T. M. P. Tait, JHEP 1009, 037 (2010).

[26] A. Pukhov, E. Boos, M. Dubinin, V. Edneral, V. Ilyin, D. Kovalenko, A. Kryukov and V. Savrin et al., hepph/9908288; A. Pukhov, hep-ph/0412191.

[27] O. J. P. Eboli and D. Zeppenfeld, Phys. Lett. B 495, 147 (2000); S. Kanemura, S. Matsumoto, T. Nabeshima and N. Okada, Phys. Rev. D 82, 055026 (2010). 